Ann. Sci. forest., 1979, 36 (4), 341-345.

\title{
Etude et mise au point d'un évaporomètre Piche enregistreur
}

\author{
P. GROSS
}

Station de Sylviculture et de Production (Bioclimatologie forestière)

Centre national de Recherches forestières, I.N.R.A.,

Champenoux, 54280 Seichamps

\begin{abstract}
Résumé
II s'agit d'un dispositif photoélectrique qui permet l'enregistrement de la hauteur d'eau dans un évaporomètre «Piche ». On suit ainsi en continu l'évaporation tout au long de la journée, et permet le fonctionnement automatique d'un poste météorologique.
\end{abstract}

Jusqu'à présent la mesure du pouvoir évaporant de l'air par la méthode de l'évaporomètre Piche nécessitait la présence d'un opérateur qui relevait périodiquement la hauteur d'eau dans le tube gradué.

Nous avons étudié un dispositif qui permet l'enregistrement suivi de la hauteur d'eau dans le « Piche » afin de suivre en continu l'évaporation tout au long de la journée et de permettre le fonctionnement automatique d'un poste météorologique.

\section{Principe de l'appareil (voir fig. 1)}

Un dispositif photoélectrique constitué d'une diode électroluminescente et d'un phototransistor fonctionnant tous deux dans le rayonnement infrarouge, détecte le bas du ménisque dans un Piche non gradué. Le phototransistor commande un motoréducteur à courant continu qui entraîne l'ensemble photoélectrique par l'intermédiaire d'une tige filetée pour que le faisceau infrarouge se situe toujours juste en dessous du bas du ménisque. Un potentiomètre couplé à la tige filetée par un réducteur (boîte de vitesse) transforme le déplacement linéaire de l'ensemble en une variation de résistance électrique qui est aisément enregistrée sur papier diagramme d'un appareil classique (enregistreur galvanométrique ou potentiométrique).

\section{Fonctionnement de l'ensemble électronique}

La diode électroluminescente est alimentée en courant par l'ensemble des résistances $R_{1}, R_{2}$ pendant la période hors gel (Piche à l'eau distillée) et par la seule résistance $R_{2}$ pendant les mois froids (1 ${ }^{\text {er }}$ novembre au 31 mars = Piche rempli d'un mé- 
FIG. 1.
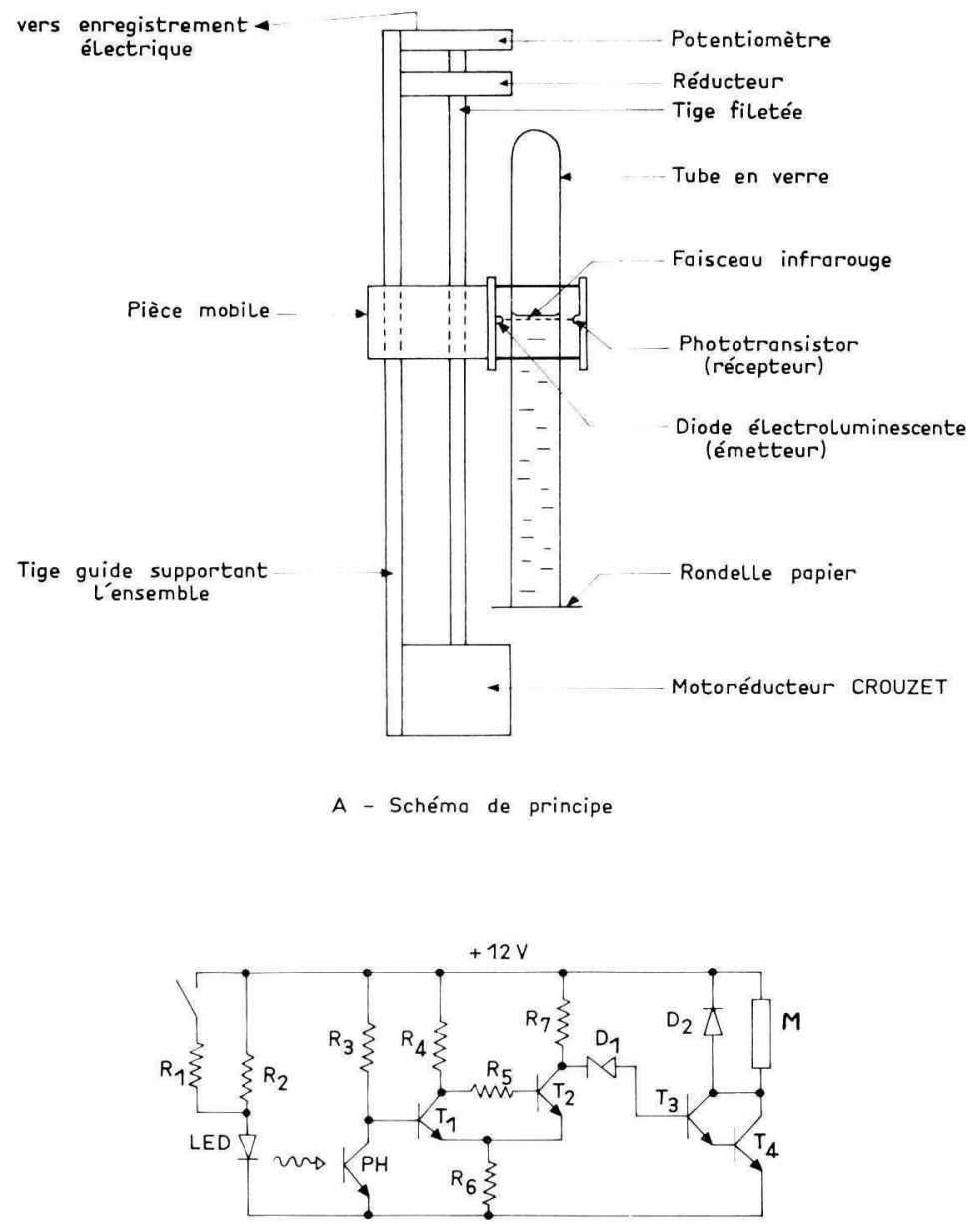

$B$ - Schémo électrique

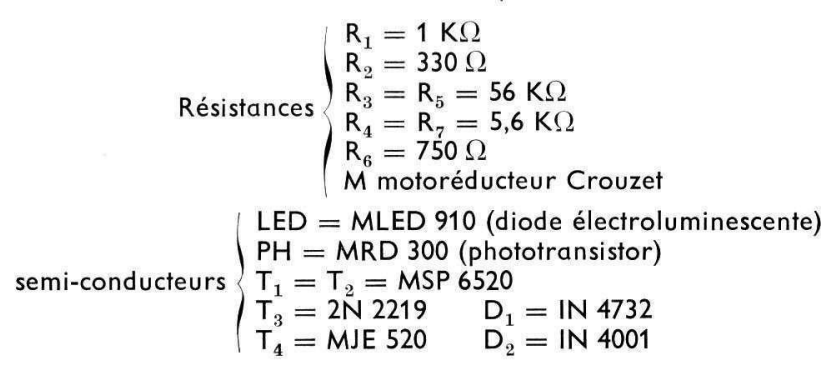

lange eau + alcool). L'eau pure absorbant plus l'infrarouge que le mélange eau + alcool : le courant traversant la diode doit en effet être plus intense dans le premier cas pour que l'énergie reçue par la phototransistor soit toujours identique. Les deux états électriques de la bascule de Schmidt (formée par les transistors $T_{1}$ ef $T_{2}$ ) sont 
déterminés par la tension aux bornes du phototransistor $\mathrm{pH}$. La commande du motoréducteur se fait par l'ensemble amplificateur Darlington $T_{3}, T_{4}$.

Au moment du remplissage du Piche, l'ensemble émetteur récepteur est ajusté de telle manière que le faisceau infrarouge traverse la partie du tube en verre non occupée par l'eau. L'énergie reçue par le phototransistor est alors très faible, $T_{1}$ est saturé *, $\mathrm{T}_{2}$ est bloqué * ; l'ensemble Darlington $\mathrm{T}_{3}, \mathrm{~T}_{4}$ est aussi saturé provoquant l'alimentation du moteur et le mouvement descendant de l'ensemble émetteur-récepteur.

Dès que le faisceau infrarouge arrive en dessous du ménisque, l'énergie reçue par le phototransistor augmente (l'ensemble tube en verre-eau formant une sorte de lentille), l'ensemble de Schmidt bascule, bloque l'ensemble Darlington provoquant ainsi l'arrêł du moteur.

L'enregistrement électrique à la suite, en continu, ne pose aucun problème.

\section{Résultats}

Le prototype mis au point en 1975 fonctionne depuis le mois de Décembre de cette même année. Durant ces deux ans la comparaison Piche « standard »/Piche enregistreur a été effectuée ; on observe une relation très linéaire (voir fig. 2) entre

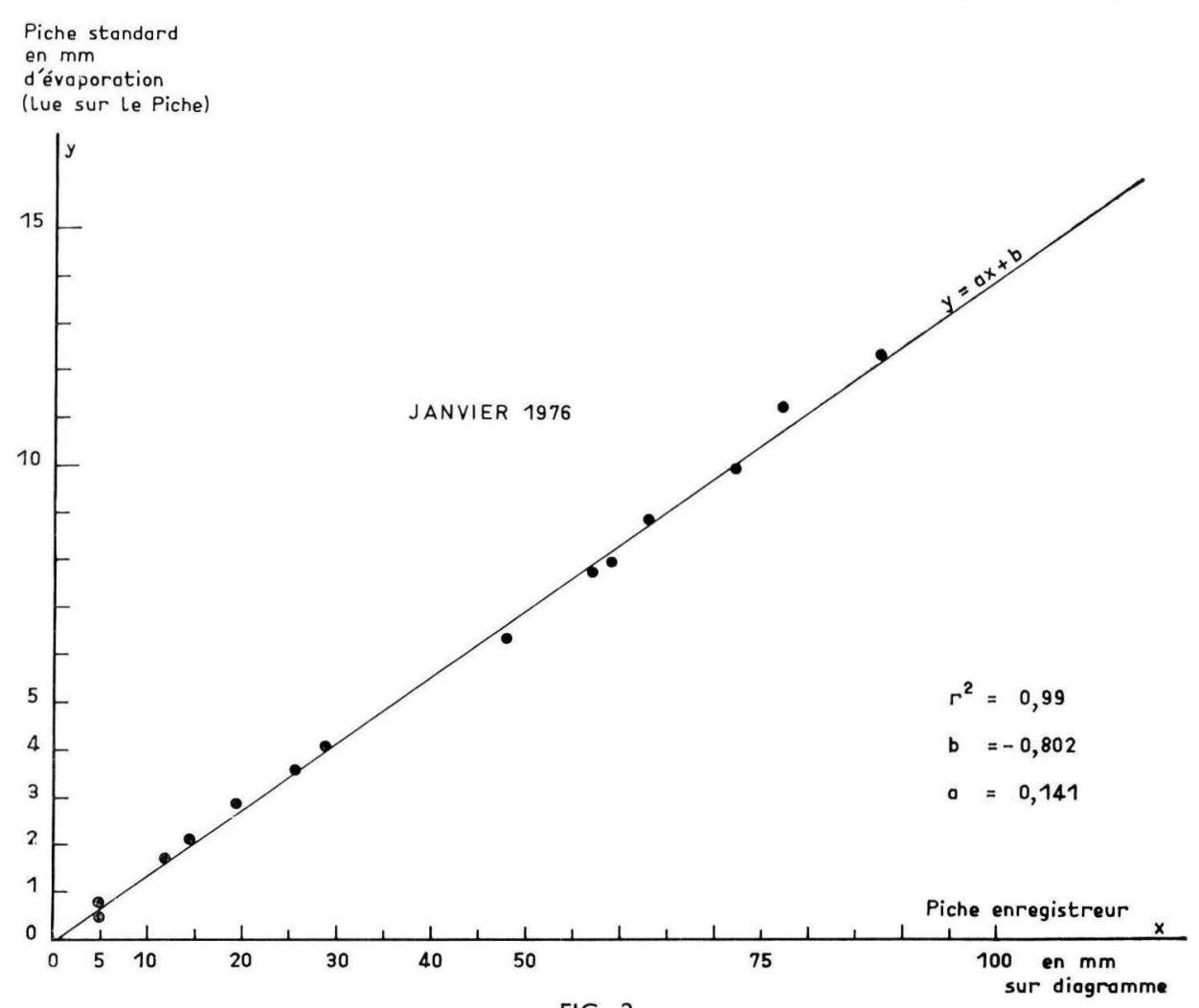

FIG. 2.

* Un transistor est dit « saturé » lorsqu'il laisse passer le courant ; dans le cas contraire il est dit bloqué. 


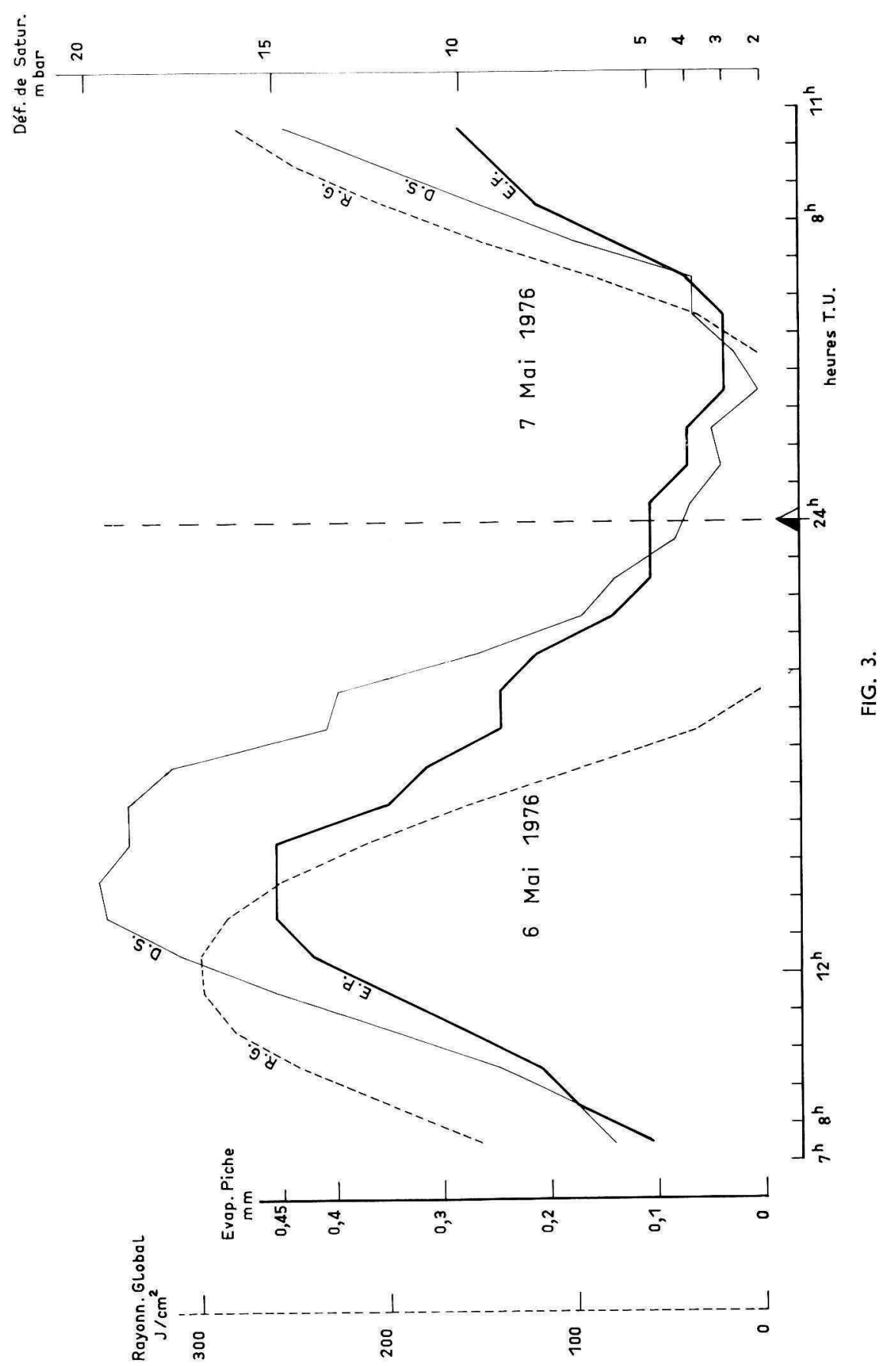


les valeurs lues par l'opérateur sur le piche "standard 》 lors des relevés météos classiques et celles données par l'enregistreur galvanométrique. Le coefficient a qui permet de passer des unes aux autres reste le même quel que soit le liquide contenu dans le Piche (eau ou eau + alcool).

La mesure de l'évaporation journalière est ainsi possible en l'absence du « préposé » aux relevés météos classiques.

Mais on peut suivre l'évaporation d'une manière beaucoup plus fine : des moyennes horaires ont été faites à partir de l'enregistreur pour de belles journées de l'été 1976 et on remarque la très bonne corrélation entre le déficit de saturation et l'évaporation mesurée par le Piche enregistreur (fig. 3).

L'appareil permet donc de pallier l'absence de relevés manuels classiques. II permet aussi l'enregistrement continu et fidèle de l'évaporation.

\section{Améliorations du prototype}

Depuis 1975, année de conception de l'appareil, plusieurs modifications ont été envisagées :

- emploi d'un micromoteur et d'un réducteur plus légers et plus compacts qui permettront de rendre l'ensemble moins encombrant,

- alimentation de la diode électroluminescente par impulsions afin de diminuer la consommation globale de l'appareil ef de le rendre autonome (alimentation par batterie),

Ces modifications ont d'ailleurs été apportées sur un dispositif s'inspirant du Piche enregistreur et qui permet de suivre l'accroissement en hauteur d'un cèdre.

Reçu pour publication en février 1979.

\section{Summary}

Study and development of a recording Piche evaporimeter

The photoelectric device allows to record the water level in a Piche evaporimeter. It is thus possible to monitor evaporation throughout the day. Furthermire it allows the automatic functioning of a meteorological station. 